\title{
Mental health economics: the Netherlands experience
}

\author{
Aileen Murphy ${ }^{1}$, Thea van Asselt ${ }^{2}$ \\ ${ }^{1}$ Department of Economics, Cork University Business School, University College Cork, Cork - Ireland \\ ${ }^{2}$ Department of Epidemiology and Department of Health Sciences, University Medical Center Groningen, University of Groningen, \\ Groningen - The Netherlands
}

Over the last 30 years there has been significant progress in economic evaluations in the mental health area. Two reviews conducted in the late 1990s, undertaken by Cabasés (1) and Evers et al (2), and a subsequent review by Roberts et al (3), were disparaging. Authors found that most mental health care interventions either had not been evaluated or were poorly evaluated $(1,2)$. Issues highlighted ranged from incomplete costs, poor quality econometric analyses and segregation of cost and health outcome information (3).

In conducting economic evaluations in the mental health field, researchers and analysts are faced with several challenges, including narrow perspectives, small sample sizes and short follow-up periods (4).

Evers et al (2) point to issues with generating evidence, many of which are affiliated with employing randomised controlled trials in the mental health area; the time horizons employed in trials/studies; transferability of results from trial settings, etc. As a result experts have urged researchers and analysts to carefully consider the choice of patient outcome, patient heterogeneity and statistical uncertainty in their data (5).

Over time the use of decision analytical modelling has also increased in the field of mental health. Good quality modelling can offer practical support for some of the aforementioned concerns $(6,7)$. It allows for synthesis of data from a variety of sources, extrapolation of information beyond trial endpoints and capturing heterogeneity across different patient groups/scenarios $(8,9)$. All of these are often necessary when interventions/policies are new and long-term evidence is scarce, as is often the case in the mental health field.

Since these reviews, the quality and quantity of economic evaluations in the mental health field have been improving, the latter often driven by governments' concerns about rising health care expenditure. Such trends are experienced in

Received: July 6, 2020

Accepted: July 8, 2020

Published online: December 14, 2020

Corresponding author:

Thea van Asselt

Department of Epidemiology and Department of Health Sciences

University Medical Center Groningen

University of Groningen

Groningen - The Netherlands

a.d.i.van.asselt@umcg.nl many jurisdictions where there is a strong tradition of economic evaluations in mental health including the UK, the USA, Australia and the Netherlands.

In 2016, the Dutch National Health Care Institute issued updated guidelines for performing economic evaluations in health care (10), replacing the earlier guidelines from 2006. Although the guidelines are mostly tailored to appraisals of drugs for reimbursement decisions, Dutch health economists tend to apply them as much as possible for all types of health care interventions, and in all fields, including mental health. The guidelines state, among other things, that the time horizon for an economic evaluation should be lifetime if possible, and outcome measures used should include Quality-Adjusted Life Years (QALYs), preferably measured using EuroQoL-5D (11). In the mental health care setting, it can be a challenge to follow these guidelines. Compared to many other clinical fields, it may prove more difficult to find evidence for efficacy of interventions in the long term, because there is less of a culture of large clinical trials. Also, the EuroQoL-5D, although its psychometric properties are in itself adequate (12), is not commonly considered a sufficiently sensitive instrument to capture the effects of mental health care interventions $(13,14)$.

Another challenge for performing economic evaluations in mental health care was posed by a shift in the Dutch health care system in 2015. For the conduct of economic evaluations, this transition, which partly entailed decentralization, hampers comparison of recent and historical data. Also, information about resource use is more dispersed than before and unit prices may vary substantially throughout the country.

Nevertheless, Dutch health economists through the years have demonstrated that performing state-of-the-art economic evaluations in the field of mental health care is feasible and does provide useful information for decision makers. Here we present focus contents of "Mental Health Economics: the Netherlands experience" showcase the quality and breadth of applied health economics in the region.

The breadth of topics addressed is already visible at first glance. The first article is on setting a research agenda for evaluating psychosocial interventions in the youth sector, followed by an article on psychomotor therapy in the rehabilitation of chronic pain patients. The third article is on improving the physical health of mentally ill patients, whereas the last article concerns the typical Dutch phenomenon of mental health professionals who are based within general practice, the so-called GP-MHPs.

The general impression emerging from these studies is that cost-effectiveness is still sometimes hard to demonstrate 
because of factors such as suboptimal responsiveness of outcome measures used, small sample size, limited follow-up and substantial drop-out. These persistent issues are not unique for the Dutch situation, but they could be, at least partly, related to the field of mental health research. Nevertheless, this should not discourage health economists from performing high-quality economic evaluations. Hopefully, increased attention for mental health globally will also further improve the conditions for economic evaluations in this area.

\section{References}

1. Cabasés JM. The cost of mental health services. Prospects for future research. Epidemiol Psichiatr Soc. 1997;6:199-208.

2. Evers S, Van Wijk A, Ament A. Economic evaluation of mental health care interventions. A review. Health Econ. 1997;6: 161-177.

3. Roberts E, Cumming J, Nelson K. A review of economic evaluations of community mental health care. Med Care Res Rev. 2005;62:503-543. doi: 10.1177/1077558705279307.

4. Byford S, McCrone P, Barrett B. Developments in the quantity and quality of economic evaluations in mental health. Curr Opin Psychiatr. 2003;16:703-707.

5. Hoch JS, Dewa CS. Lessons from trial-based cost-effectiveness analyses of mental health interventions. Pharmacoeconomics. 2007; 25:807-816.

6. Brennan A, Akehurst R. Modelling in health economic evaluation. Pharmacoeconomics. 2000;17:445-459.
7. Evers S, Salvador-Carulla L, Halsteinli V, McDaid D. Implementing mental health economic evaluation evidence: building a bridge between theory and practice. J Ment Health. 2007;16:223-241. doi: 10.1080/09638230701279881.

8. Sheldon TA. Problems of using modelling in the economic evaluation of health care. Health Econ. 1996;5:1-11.

9. Drummond M, Sculpher MJ, Torrance GW, O'Brien BJ, Stoddart GL. Methods for the economic evaluation of health care programmes. 3rd ed. Oxford: Oxford University Press; 2007.

10. Dutch Healthcare Institute. Guideline for economic evaluations in Healthcare. Diemen, 2016. https://english.zorginstituutnederland.nl/publications/reports/2016/06/16/guideline-foreconomic-evaluations-in-healthcare. Accessed June 15, 2020.

11. Herdman M, Gudex C, Lloyd A, et al. Development and preliminary testing of the new five-level version of EQ-5D (EQ-5D-5L). Qual Life Res. 2011 Dec;20:1727-1736.

12. Mulhern B, Mukuria $C$, Barkham M, et al. Using generic preference-based measures in mental health: psychometric validity of the EQ-5D and SF-6D. Br J Psychiatry. 2014 Sep;205:236-243. doi: 10.1192/bjp.bp.112.122283. [Epub 2014 May 22].

13. Brazier J, Connell J, Papaioannou D, et al. A systematic review, psychometric analysis and qualitative assessment of generic preference-based measures of health in mental health populations and the estimation of mapping functions from widely used specific measures. Health Technol Assess. 2014;18:1-188. doi: 10.3310/hta18340.

14. Connell J, O'Cathain A, Brazier J. Measuring quality of life in mental health: are we asking the right questions? Soc Sci Med. 2014;120:12-20. doi: 10.1016/j.socscimed.2014.08.026. [Epub 2014 Aug 20]. 